\title{
On concepts and methods in horizon scanning: Lessons from initiating policy dialogues on emerging issues
}

\author{
Effie Amanatidou ${ }^{1, *}$, Maurits Butter ${ }^{2}$, Vicente Carabias ${ }^{3}$, \\ Totti Könnölä ${ }^{4}$, Miriam Leis ${ }^{5}$, Ozcan Saritas ${ }^{1}$, Petra Schaper-Rinkel ${ }^{6}$ \\ and Victor van $\mathrm{Rij}^{7}$ \\ ${ }^{I}$ Manchester Institute of Innovation Research, MBS, Harold Hankins Building, \\ University of Manchester, Manchester, M13 9PL, UK \\ ${ }^{2}$ TNO, Wassenaarseweg 56, 2333 AL Leiden, The Netherlands \\ ${ }^{3} E U$ DG JRC-IPTS, Seville, Spain, and ZHAW Zurich University of Applied Sciences, \\ Institute of Sustainable Development, Postfach 805, CH-8401 Winterthur, Switzerland \\ ${ }^{4}$ Impetu Solutions, Victor Andrés Belaunde, 36-4C, 28016 Madrid, Spain \\ ${ }^{5}$ Editage/Cactus Communications Inc., C-912 BSEL Tech Park, Sector 30A, Vashi, \\ New Mumbai 400 705, India \\ ${ }^{6}$ Austrian Institute of Technology, Donau-City-Straße 1, 1220 Vienna, Austria \\ ${ }^{7}$ Advisory Council for Science and Technology Policy, Javastraat 42, 2585 AP The Hague, \\ The Netherlands \\ *Corresponding author. Email: amana@otenet.gr.
}

Future-oriented technology analysis methods can play a significant role in enabling early warning signal detection and pro-active policy action which will help to better prepare policy- and decision-makers in today's complex and inter-dependent environments. This paper analyses the use of different horizon scanning approaches and methods as applied in the Scanning for Emerging Science and Technology Issues project. A comparative analysis is provided as well as a brief evaluation the needs of policy-makers if they are to identify areas in which policy needs to be formulated. This paper suggests that the selection of the best scanning approaches and methods is subject to contextual and content issues. At the same time, there are certain issues which characterise horizon scanning processes, methods and results that should be kept in mind by both practitioners and policy-makers.

Keywords: emerging science and technology issues; early warning signals; weak signals; horizon scanning; policy support.

\section{Introduction}

The 2000s have witnessed increasing complexities in societies. Although the world has improved for some people, the vast majority of people appear to be vulnerable to social and economic instability and hostility due to the economic recession, lack of fresh water, shortages of food and energy, climate change, regional conflicts, and population movements. At the same time, the new global context suggests increased financial, trade and investment flows leading to high levels of interconnectedness. It is also observed that the current challenges, (such as the above), facing societies are highly complex with uncertain and far-reaching implications. Within this context, it is crucial to inform policy- and decision-makers about new and future opportunities as well as threats and have them prepared (or make them aware) for drastic changes and surprises/shocks. 
A relevant tool for this purpose is horizon scanning defined as:

... the systematic examination of potential (future) problems, threats, opportunities and likely future developments, including those at the margins of current thinking and planning. Horizon scanning may explore novel and unexpected issues, as well as persistent problems, trends and weak signal. (Van Rij 2010a, 2010b)

Horizon scanning may also take place in specific policy domains (Botterhuis et al. 2010).

Horizon scanning is generally seen as an instrument with two main functions: the alerting and the creative functions. The alerting function helps policy-makers to anticipate emerging issues better and earlier, while the creative function enables the reassembly of issues or the creation of new emerging issues on the basis of the analysis and integration of scan data. In this way horizon scanning contributes to opening up new policy options or testing developed policies in relation to their resilience to unknown, but plausible, new emerging issues. This is the case for example with the horizon scanning services in the UK. ${ }^{1}$ Horizon scanning also fulfils similar functions with scenario building. It may even be used as a highly evidence-based source to provide plausible discontinuities in the development of scenarios.

Recently, horizon scanning activities have been applied at the national level to inform national foresight exercises, thus providing a more evidence-based approach to research and innovation policy (Georghiou 2007). The main focus of this activity has been to address new and emerging technological areas that may have an impact on social, economic, environmental and political developments. National horizon scanning activities have been carried out as in the UK, the Netherlands and Denmark (Van Rij 2010a).

A special national horizon scanning activity is taking place in Singapore under the Risk Assessment and Horizon Scanning programme which led to the development of a semi-automatic system that provides continuous end-to-end capabilities to collect and classify data, analyse and understand relationships, and anticipate as well as discover emerging issues that could have a strategic impact on Singapore. It combines tools, methods and networks for effective scanning and analysis. ${ }^{2}$ Horizon scanning places great emphasis on building a holistic framework whereby the output of scanning can be effectively incorporated into vision building and strategic planning.

The present paper draws on the experiences from the seventh European Framework Programme horizon scanning project Scanning for Emerging Science and Technology Issues (SESTI). ${ }^{3}$ During the project the present authors developed an approach to organise and implement horizon scanning processes effectively in order to integrate the diverse information needs of policy-makers about emerging issues. This paper is structured as follows: Section 2 introduces the main definitions, as well as the different scanning approaches and scanning tools that were applied in the SESTI project. Section 3 provides a comparative analysis of the two scanning approaches and the associated tools that were used in SESTI. Building on the advantages and disadvantages of each tool and method, Section 4 evaluates the different approaches and tools based on evaluation criteria reflecting the information needs of policy-makers about new and future opportunities and threats. Finally, Section 5 draws conclusions on how horizon scanning can be used to improve policy formulation and dialogue.

\section{Defining and clarifying concepts}

\subsection{Weak signals alongside emerging issues and wild cards}

Horizon scanning implies a search process, which is extended at the margins of the known environment and possibly beyond it (Loveridge 2009). Horizon scanning aims to identify emerging issues and events which may present themselves as threats or opportunities for society and policy. Additionally, in this special issue Könnölä et al. (2012) regard horizon scanning as:

... a creative process of collective sense-making by way of collecting and synthesising observations that hold potential for the formulation of pertinent future developments and the derivation of actionable implications on decision-making.

Horizon scanning typically builds on concepts such as weak or early warning signals within the framework of political discourse and decision-making. Early warning signals are the first important indications of a change. These signals are often difficult to spot. Therefore they are usually referred at as faint or weak signals.

These terms are closely connected, if not confused, with the concepts of emerging issues and wild cards. Table 1 provides the definitions of these concepts, shedding some light on their differences. While uncertainly and incompleteness characterise weak signals, it is their sudden nature and high impact that mark wild cards. On the other hand, the concept of an emerging issue refers to a higher level of abstraction in the sense that an issue emerges when several weak signals combine together, based on interlinked possible impacts.

\subsection{Exploratory and issue-centred scanning}

There are different approaches which underpin the scanning process. One way of approaching scanning is to differentiate between exploratory and issue-centred scanning.

The exploratory scanning approach concentrates on assembling potential emerging issues from a wide variety of data from different signal sources, while the issue-centred approach concentrates on identifying core documents (primary signals) that describe substantial 
Table 1. Summary of concepts applied in horizon scanning

\begin{tabular}{|c|c|}
\hline Concept & Description \\
\hline Weak signals & $\begin{array}{l}\text { Citing from Igor Ansoff, Hiltunen provides a definition of weak signals as: 'warnings (external or internal), events and } \\
\text { developments that are still too incomplete to permit an accurate estimation of their impact and/or to determine their } \\
\text { complete responses' (Hiltunen 2008a). Weak signals can be further divided to physical or social signals (Uskali 2005) }\end{array}$ \\
\hline Wild cards & $\begin{array}{l}\text { Wild cards are events with a surprising character, a low probability and a high impact (Van Rij 2011). These events tend } \\
\text { to alter the fundamentals, and create new trajectories which can then create a new basis for additional challenges and } \\
\text { opportunities that most stakeholders may not have previously considered or prepared for (Saritas and Smith 2011). } \\
\text { Wild cards represent the occurrence of a singular (historically original), sudden (abrupt, fast), surprising (unexpected) and } \\
\text { shattering (serious, severe) event that come out of the blue and therefore present a significant foresight challenge both } \\
\text { for policy makers and researchers (Mendonça et al. 2009) }\end{array}$ \\
\hline Emerging issues & $\begin{array}{l}\text { Beyond horizon scanning the concept of emerging issues is also used in the area of risk assessment (Marvin et al. 2009), in } \\
\text { the field of environmental issues (Sutherland et al. 2010) and in organisations in their environmental scanning (Morrison } \\
\text { 1992). It comes close to the concept of future narratives as used by Van der Steen (2008) in his political discourse } \\
\text { analysis. Van der Steen (2008) sees future narratives as 'stories about what the future, or possible futures, may or will } \\
\text { (depending on the narrative) look like and that connect these possible futures to current issues for political debate (the } \\
\text { discourse)'. Recent horizon scans of the UK, Netherlands and Denmark show us many examples of smaller and larger } \\
\text { potential emerging issues. They usually describe a future narrative or mini scenario that, according to the cited author or } \\
\text { source, should be taken into account by policymakers, society and/or research. Recent examples have been analysed by } \\
\text { Boden et al. (2010) }\end{array}$ \\
\hline $\begin{array}{l}\text { Discourse and } \\
\text { decision-making }\end{array}$ & $\begin{array}{l}\text { Political discourse refers to the continuous communicative debate that takes place in the political arena. This debate is } \\
\text { unique for human beings with their ability of speech and writing (Chilton 2004; Van Dijk 2002) and nowadays audiovisual } \\
\text { presentations and computer simulations. The outcome of a political discourse is partly dependent on the contents and } \\
\text { shape of the communicative expressions of the participants their cognition in wide sense as well as the power relationships } \\
\text { between the participants (Chilton 2004; Van Dijk 2002). Issue description and the early warning signals around them are } \\
\text { communicative expressions that will eventually have to find their place in this policy discourse in order to be relevant }\end{array}$ \\
\hline
\end{tabular}

parts of potential issues. In the first approach emerging issues are identified and described by processing information from different sources, while in the issue-centred approach the (preliminary) descriptions of issues are used as a core to identify potential additional signals that could either confirm or deny the real emergence of the issue.

2.2.1 Exploratory scanning. In exploratory or curiositydriven scanning the analyst, with no specific framework or any potential emerging issues in mind, searches for information about new developments. This can be done by using automated tools such as news alerts and feeds, Google trends as well as automated text-mining. However, the novelty, quality and reliability of the information that can be retrieved in such a totally focus-free and open way is highly questionable. A refined method of exploratory scanning is offered by applying a more bottom-up approach where the analyst examines the observations and topics that different (independent) scanning communities focus upon, and how this information is transformed into potential emerging issues that can be interesting and useful for decision- and policy-makers.

The aim of the exploratory scanning approach is to identify a long list of signals that are precursors for emerging issues, only demarcated by the policy domain selected (e.g. healthcare or energy). The raw material may be different observations about topics such as scientific and technological developments within various areas, and may be obtained from research papers, laboratory results, dissemination about new theories, experiments, prototypes etc. Automated text-mining tools as well as databases that allow for tagging and categorisation can help with clustering individual observations. Thus, one may end up with different sets of individual observations that could be related to each other under certain headings such as: new developments and funding aimed at minimising the negative health effects of human ageing or understanding, utilising and improving the working of the human brain, or seeking and providing new possibilities for new energy technologies and infrastructures. However, within a real bottom-up approach, the clusters are still open and are determined after a sufficiently large number of observations have been collected.

The exploratory scanning approach includes various steps:

- Setting a heuristic search profile: Based on the domain of the audience a search profile should be set in order to obtain a more focused search. However, this search profile is only slightly focused, using keywords (or operators) such as: innovation, emerging, issues, impact, change, future, emerging, promising, threatening, solutions, discoveries, problems, crisis, tensions, growth, breakthroughs, breakdowns, or new insights in combination with the domain demarcating keywords.

- Broad scanning for signals: The preliminary identification of signals can use various sources, like wikis, Twitter, and other internet sources such as blog reports, literature consultation, but also conference 
proceedings. Additional information and leads are obtained by participative approaches, for instance setting up a (SESTI) wiki, evoking Twitter activity around SESTI and more traditional methods such as a survey. The vast amount of data coming from these sources can be analysed in terms of potential signals of change, where text-mining can be used as a tool for analysis. It is important is that the search profile is focused on the selection of sources to be used (wiki, experts invited, Twitter accounts, blogs and websites, conferences, reports and papers).

- Preliminary assessment and selection: The selection of key signals from the vast amount of data coming from the various sources is done by experts. This assessment refers to the potential underlying emerging issue and not to the individual signal. Criteria that can be used include: plausibility (e.g. trustworthiness of source), potential impact (structural, interests at stake), novelty (lack of policy) and precursor's weight (strength of signal to the issue).

- Clustering of signals: The long list of signals is then clustered, using potential emerging issues as a clustering mechanism. Text-mining can also be used to identify clusters.

2.2.2 Issue-centred scanning. Whereas the above, bottom-up approach can be considered as hypothesisgenerating, the issue-centred approach can be rather seen as evaluating a hypothesis (the hypothesis of proposed emerging issues). The issue-centred approach starts from the wide range of existing and potential emerging issues (hypotheses) and searches for weak signals in order to strengthen or question the specific hypotheses, i.e. reinforce the hypothesised emerging issue or reveal changes, modifications and disruptions of existing emerging issues in a significant way.

Automatic tools are seen as methods to support the scanning for potentially highly important weak signals and to monitor and trace the weak signals and their sources.

As a starting point a frame of reference is conceptualised for the chosen policy domains. Signals are then sought that give a full or substantial future narrative with high impact for a certain policy level. We refer to these signals as primary signals. They are usually articles or presentations by an author or group of authors, which describe a future narrative with a foreseen great impact on future science, society or economy in combination with a need for present-day (policy) decisions to prevent, mitigate or stimulate the foreseen impact. The storyline is based on their observations of breakthroughs, discoveries and/or events which facilitate a single or multiple future storyline leading to the impact. The story often contains some recommendations, or at least suggestions, on the way the positive impact could be reached or the negative impact could be prevented.

Only items (articles, websites and videos) with more or less full storylines that connect factual findings or plausible assumptions in a logical way with a foreseen future high impact are considered. These high impact items are supported by reliable sources such as: scientific articles, statistical analysis, trend reports and foresight exercises. The storyline may address conflicts or common interests as well as emotional, ethical and legal aspects. The storyline usually gives implicit or explicit elements that could be used as indicators for the realisation of the storyline. These elements give clues for further scanning of signals that may support or deny the possibility that the story will occur (the signals are known as secondary signals).

In a second round additional material is sought, that may confirm or oppose the selected storylines. If the secondary signals have manifested themselves in the period before the scanning was done, then they can be added to the description of the issues. In most cases it will also be necessary to track the secondary signals in the near future to see whether the issue that was described in the primary signals really evolves or dissolves. Even when the primary signal is followed by many positive secondary signals and attracts a lot of policy attention the issue may suddenly head in a completely undirected way because of the occurrence of wild cards or countervailing issues. It should also be clear that issue-centred scanning does not predict issues. Rather it provides tools to alert for potential impact-rich issues that need policy attention.

In order to identify and assess the primary signals, similar criteria were applied as for the bottom-up exploratory scanning presented above: described impact, desirability, factual basis, plausibility, novelty to policy-makers, interests at stake, emotional aspects and critical aspects (if the issue appeals or concerns emotional or ethical legal aspects), and changeability (if the story or its impact can be altered by human action and how the issue relates to present-day decision-making and action).

Rather than being antagonistic, the two approaches should be seen as complementary (see Fig. 1). Exploratory scanning mainly refers to the first scanning phase (identification of weak signals) while issue-centred scanning spans throughout phases 1 and 2 of the SESTI scanning process (cf. Section 3.1).

\subsection{A variety of sources and scanning tools}

Both of the above approaches used the internet as their main source of information. This made it possible not only to search for signals in officially published documents and reports but also to exploit relevant discussions in blogs and micro-blogs (as Twitter), wikis and websites which provide non-textual information as videos and audios. In addition, experts and policy-makers were called upon to enrich, validate and discuss the scanning results. 


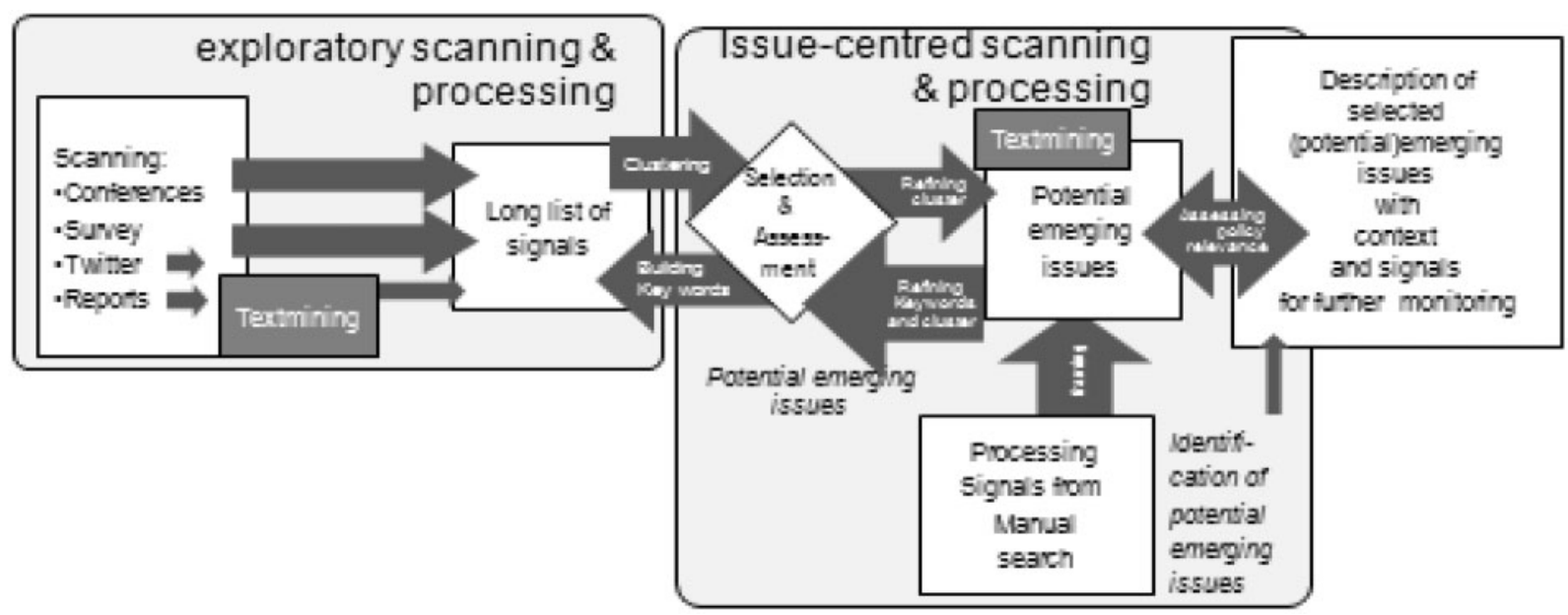

Figure 1.

In the SESTI project the following tools were used for the scanning: ${ }^{4}$

- web-based search engines as Google, Google News Timeline, ${ }^{5}$ Google Insight $^{6}$ and Bing ${ }^{7}$

- expert review and survey

- visits to conferences and seminars

- a special SESTI wiki to evoke contributions to the scanning process

- active use of blogging and micro-blogging (Twitter $)^{8}$

- text-mining

- expert/stakeholder workshops

Based on the experience of SESTI, the scanning tools can be grouped with regards to the level of participation, and the means of processing information. Some of the methods are suitable for obtaining specific information like expert reviews, surveys and visits to conferences and seminars while other tools like the initiation of a wiki and the active use of blogs and micro-blogging can encourage wider participation and dialogue. Search engines and text-mining can be applied without the involvement of wider groups outside the project core team (nonparticipatory).

The second way of grouping involves the identification, processing and analysis of weak signals and emerging issues with the help of various levels of automation. These methods can be mapped within a methodological framework (see Fig. 2).

The focused expert review was mainly based on internet scanning with the use of search engines. It was performed by professional scanners. Internet scanning makes it possible to search for signals that can be traced in written sources but also in audiovisual material. The scanners were assisted by expert panels (expert review) that reviewed the raw observations of the searches. These raw observations contained issue-focused excerpts from written, audiovisual and other sources. Written sources
Participatory methods

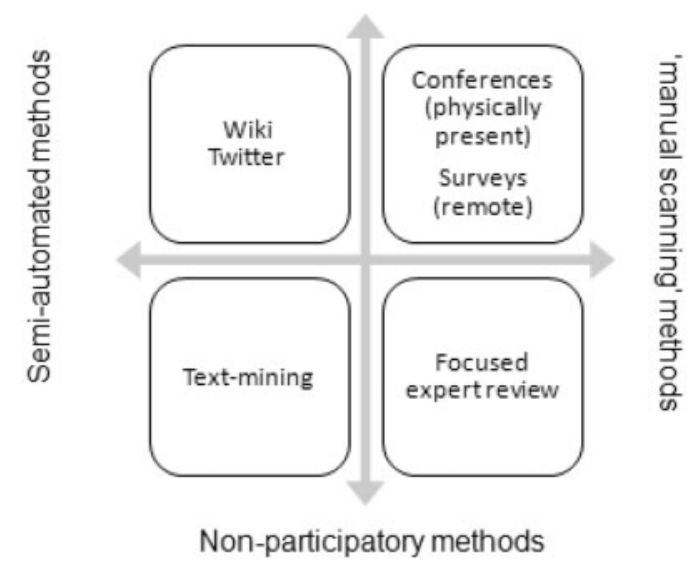

Figure 2.

included scientific and non-scientific articles in e-journals and newspapers, but also in wikis, specialised websites, specialised web-blogs, policy papers and reports. Audiovisual and other sources were audio and video recordings of interviews, but also video documentaries, reports and databases. A focused expert review supported by internet scanning has the advantage that it can use any available source and tool that is on the internet.

Wikis are a website-based technology for mass collaborative authoring. Looking at the considerable success of Wikipedia, a wiki could be an interesting source from which to collect signals on emerging issues. It can be a platform for collecting information about signals and issues, as well as being a place to facilitate expert discussions. Wikis are ideally suited for collaborative learning and have the potential to foster participation, encourage collaboration, and lead to a rapidly growing content. 
Many of the sophisticated community annotation systems that are currently used in biology are based on the wiki concept (Mons et al. 2008; Stein 2008). In the SESTI project an attempt was made to set up a wiki that specialised in collecting voluntary descriptions from many authors on new emerging issues for science and technology.

The attempt was not as successful as had been hoped because most of the contributors did not want to spend time writing new full-length articles on issues that had already been described elsewhere. Thus, people tended to send web links rather than writing contributions. This was one of the reasons why the wiki was abandoned at an early stage and an active Twitter account was started to collect new links, which was successful in this respect. It may be concluded that the functionality of an active wiki may easily be caught by active twittering. It is important to note that, for the wiki as well as for Twitter, contributions can only be made by experts who are active in these types of media.

Within the SESTI project, a survey was run asking various experts about new emerging issues and signals that they perceived. This method has certain limitations. First, the selection of the experts in the field could bias the outcomes. Respondents may be inclined to contribute their personal interests rather than results from open thinking (even with ideas which compete with their own interests). A second aspect is that a survey yields limited information and is bound to the moment in which it is executed. Thus, there is a limited possibility for retrieving new information. However, a statement made by an expert that an issue is emerging can also be seen as a signal. Based on the SESTI experience, an expert survey should be regarded as an additional tool with which to evaluate the issues which are identified, rather than being used to obtain original signals.

A similar conclusion can also be drawn from attending conferences. As conferences are often more focused on new ideas, the assessment of discussions can be an interesting way in which to collect signals. Conferences may also be particularly interesting for making contact with future-oriented experts as well as policy-makers in the relevant domains.

A new way of collecting signals is through active blogging and micro-blogging (Twitter). Twitter is an internet social-network and micro-blogging platform with both mass and interpersonal communication features for sharing 140-character messages (known as tweets), with other users (known as followers). A major advantage of Twitter is that it is fast and information often appears on Twitter before it is reported in conventional news formats (e.g. TV, radio and print media). Information about new developments and also societal happenings can be obtained, even in real time. This can enable faster and timelier assessments and thus earlier detection of events, changes and possible weak signals. Experts often tweet about new trends and issues and also mention important information from blogs and news websites. However, an approach to processing the information is needed, as the signals can very quickly amount to thousands of entries. The SESTI experience with Twitter shows that, in principle, it is an interesting way of collecting signals, if the right information is filtered. It is also crucial to select trusted and reliable twitterers (Pang 2010).

Text-mining enables large amounts of textual information to be assessed with specialised software. Within the SESTI project, a number of foresight reports in the field of energy and healthcare were processed with text-mining. The experience showed that text-mining has a fundamental flaw when identifying signals. An essential criterion for the signals to be identified is that they are unknown. Text-mining uses a statistical routine to identify parts of text that are frequently mentioned. This means that any issues mentioned in these texts are relatively known. More sophisticated software is needed to incorporate the functionalities that are needed for finding new signals and emerging issues. Currently text-mining is especially useful to identify networks and clusters of phrases within huge data sets, but less useful for identifying new signals and issues. The disadvantage is that the publicly available text-mining software is not yet useful for analysing audiovisual material.

\section{Comparative analysis of tools for exploratory and issue-centred scanning}

In order to place the analysis of the different approaches and tools that were used in their correct context, the main phases of the SESTI scanning process should first be clarified.

\subsection{SESTI scanning process}

Three main phases can be distinguished, as practiced in the SESTI project. Phase 1 relates to the identification of weak signals. Emerging issues are usually formulated on the basis of searches in different sources and expert interviews. These searches and interviews lead to a selection of more or less full descriptions of potential emerging issues (future narratives) and also a body of additional signals that are considered to be indicative of the start or development of their emergence.

Phase 2 relates to the processing of weak signals. This phase consists of the following main steps:

Step 1 Selection of the broader area where emerging issues will be examined.

Step 2 Clustering of weak signals.

Step 3 Assessing the significance of clustered weak signals.

Step 4 Framing the connected weak signals into clustered topics. 
Step 5 Tentative modelling of emerging issues into possible emerging issues.

Step 6 Identification of significant emerging issues.

Phase 3 is devoted to the analysis and interpretation of emerging issues with relevance for policy-making. Policy-makers interviewed at the start of the SESTI project noted that it is important that the analysis of emerging issues considers the possibilities to manage emerging issues while also identifying the associated policy implications. As has been highlighted, a proper assessment of weak signals should be translated into policy recommendations. Thus, the last phase of the scanning process should be devoted to this particular task. In the SESTI project this task was complemented by workshops which provided space for discussing the findings on emerging issues with experts as well as with policy-makers in order to draw conclusions about the implications for policy.

\subsection{Comparative analysis of methods and tools applied during the SESTI project}

The focused expert review could be used for the entire scanning process. Within the issue-centred approach it is a very useful tool with which to identify potential emerging issues in a fast and cost-efficient manner. It also enables the identification of potential secondary signals that can be used to contextualise these issues and to monitor their further development. Database tools that are connected to search engines such as Google News Timeline, Google Insight, Web of Science, ${ }^{9}$ or Gapminder ${ }^{10}$ are very helpful for this purpose. Another advantage of internet scanning is that it uses all available sources of information on the internet (including audiovisual information) as well as web tools such as those mentioned above. In addition, internet scanners can use web-based translation facilities to overcome language barriers. The restriction is the fact that professional scanners may have biases in their searches and interpretation of findings. Teams of scanners with different backgrounds would help to overcome this pitfall.

The results of the scanning can also be a starting point for taking into account the areas of unknown unknowns by spotting gaps in the scan picture and by linking unconnected areas. That is about raising the awareness of non-knowledge (areas beyond what we know we do not know). Taking into account the plurality of perspectives on non-knowledge can serve as a basis on which to establish transparent and pluralistic processes for exploring scientific non-knowledge (Böschen et al. 2010).

The focused expert review can also make use of all the tools for the identification of signals (phase 1). In the SESTI project online tools and especially web 2.0 tools were used in different ways. The tool that turned out to be less useful was the SESTI wiki. The reluctance of the community to contribute made it difficult to use this tool in any of the scanning phases.

Wikis and surveys are participative methods, and thus the representativeness, level of expertise, and commitment of participants are critical factors for success. The survey conducted in the SESTI project was also confronted with the limited time and visibility of the contributors. Although a wide variety of potential participants was addressed, the results showed that the topics were often either very general or very specific. The signals collected through the survey often led to websites and articles that were already available from other sources. This makes that specific tool less appropriate for the identification phase.

However, expert surveys can be quite useful in the processing and analysis phases where there is an explicit focus on certain fields and issues. Comparing the SESTI experience with experiences from other horizon scanning processes, it seems that surveys are especially useful when the fields are specified and the scanning starts from well-defined fields such as energy, conservation or science and policy (cf. Czaplicka-Kolarz et al. 2009; Smith et al. 2010; Sutherland et al. 2010; 2011). Focusing on specific fields, surveys can deliver additional information on various side aspects related to the core issue.

The use of Twitter could be useful for the identification of signals and issues in multiple ways. Twitter can be used as a source for signals and for tracing the way in which signals (especially in regard to potential scientific breakthroughs) are spread and taken up by different communities. Twitter can also be used as an interactive tool. However, online software tools for clustering Twitter tweets are necessary, especially for the processing and analysis phases, but these have not yet been developed. With the availability of such tools, Twitter could allow a continuing clustering of signals related to emerging issues.

Policy-makers who were interviewed within the SESTI project noted the importance of methods that allow for identifying the connections, clustering of signals and the stakeholders behind them. To obtain this information (micro-) blogs are becoming interesting tools with which to analyse which communities have taken up specific issues and how they relate those issues to other issues. The novelty of weak signals as well as rising ethical, legal, societal or cultural issues, and also possible implications, could also be traced.

Twitter was also used to obtain data for text-mining. However, with downloading and processing data from Twitter one of the huge advantages of Twitter was lost: the ability to trace how signals evolve over time. In general, however, the potential of Twitter to become a main tool for retrieving future-oriented information is high as colleagues, scientists and other futurists consider future analysts to be their most valuable source for weak signals (Hiltunen 2008b).

Overall, micro-blogging is one of the social web tools that could change how science is perceived and how 
scientists communicate about controversial topics in science. Today it is used only by a small minority. For example, analysing and tracing Twitter tweets and the related blog posts can be used to see how publications in high-ranking journals are commented on or criticised via Twitter. For the scientists in the labs these developments cause many problems because:

...the speed of communication is ahead of the sheer time needed to think and get in the lab and work. (Mandavilli 2011)

The blogosphere in general is expanding at an unprecedented speed. With a better understanding of the blogosphere it would be possible to develop tools to use collective wisdom to scan future-related signals and emerging issues. One important opportunity in this process is to use web 2.0 tools in a participatory way to involve interesting potential contributors (in particular a broad variety of experts) and the other opportunity is to use them as fast expanding sources of structured information by clustering them. Clustering blog sites presents new challenges for information science (Agarwal et al. 2010) as no tools are yet available. In addition, there is also the challenge of how to address and involve many individual contributors as well as to address a huge variety of specific communities and contributors. Until these challenges are met, the tools of the social web will remain mainly as additional tools which can be used by experts when analysing issues.

Conferences may not primarily be interesting as places where one can become aware of new signals as this information may be obtained sooner in other places. However, they are useful for discussing with future-oriented experts as well as with policy-makers the validity of hypotheses on specific emerging issues or their novelty, plausibility or impact. This is particularly relevant for the analysis and interpretation phase.

Text-mining, as noted above, needs more sophisticated tools to capture the emerging and novel nature of weak signals. However, text-mining is useful in the processing phase as it identifies networks and clusters of concepts and phrases within huge data sets. However, certain aspects of text-mining need to be considered. Today's text-mining software cannot be seen as a fully automated system. The large lists of outcomes need to be processed and an expert needs to fine tune the underlying procedure (e.g. by adapting the search heuristics). A first assessment of the tools used in the scanning process in the SESTI project is given in Table 2 .

\subsection{Policy workshops and policy discourse}

Specific reference should also be made to the policy workshops that were conducted as part of the SESTI project. These particularly helped the third phase of the scanning process (analysing and interpreting the relevant implications of emerging issues for policy-making).
Table 2. Comparison of tools used in scanning process (rate of appropriateness and usefulness: low, medium, high)

\begin{tabular}{llll}
\hline & $\begin{array}{l}\text { Phase 1: } \\
\text { Identification } \\
\text { of weak signals }\end{array}$ & $\begin{array}{l}\text { Phase 2: } \\
\text { Processing of } \\
\text { weak signals }\end{array}$ & $\begin{array}{l}\text { Phase 3: } \\
\text { Analysis and } \\
\text { interpretation }\end{array}$ \\
\hline Focused expert review & High & High & High \\
Wiki & Low & Low & Low \\
Twitter & High & Low & Low \\
Surveys & Low & High & High \\
Conferences & Low & Medium & High \\
Text-mining & Low & Medium & Medium \\
\hline
\end{tabular}

Three thematic workshops on cognitive enhancement, energy and health were organised. The participants were policy-makers and experts with a broad view of the domains being considered. Analytical presentations were made on the selected emerging issues in the different domains. These triggered the discussions that followed. After discussing the issues participants were asked to vote on the terms of the perceived impact, plausibility, novelty, policy relevance and strength of each of the emerging issues. Although policy-makers were underrepresented in these workshops, the results gave a clearer idea of the relative importance of the issues and the way in which they could be assessed.

Although some issues were assessed as very important in all aspects, policy-makers expressed doubts about whether they would be taken into further consideration because they were not sufficiently aligned with the policy agendas. Linkages to issues that were already on the radar of policy-makers were considered helpful for improving the relevance and usefulness of the scanning outcomes. The workshop experience also showed that even though topics or issues may already be known, they can still be considered to be emerging if they are considered to be of relevance for the future but have not been sufficiently taken into consideration by policy and society thus far.

Additionally, the timing of bringing up signals or emerging issues could be a crucial factor for their further consideration in the policy-making arena. Timing seems to be a general challenge with early signal analysis. Due to the novelty of issues the evidence basis at the beginning is rather weak while the impact may be tremendous. Thus, their dissemination may encounter scepticism or be totally ignored. Once evidence is built up the claim for considering them becomes more accepted and recognised. However, the signal will no longer be novel and the ability to prevent negative impacts may be lost. As the signal starts to have visible negative impacts on its environment, possible controversies and disruptions may emerge alongside a general frustration about the lost opportunity to be informed earlier about the potential upcoming developments. This forms a difficult challenge for scanners who 
want to be successful by giving the right alerts to policy-makers at the right time in order not to over-occupy their busy agendas.

Apart from timing, there are also challenges in bringing forward a potential emerging issue or early signal to policy-makers. Policy-makers may face barriers to taking the results of horizon scans on board as they may be contradicted by vested interests. This may particularly be the case with issues which include controversies or require coordination and collaboration across different and segmented policies. In the cases where conflicts may arise even small investments in examining the issues may be blocked.

Overall, potential wild cards and emerging issues, including their early warning signals, have to fight for attention in the political discourse except for situations in which they fit or are strongly connected into the present-day discourse and provided they are not against the interests of powerful lobbies. Notwithstanding, a strong future narrative is able to draw the attention of the participants and agenda setters. But, there is not only competition among future narratives but also between future narratives and present-day competing events, which may include huge-impact wild cards like the surprisingly strong earthquake which occurred in Japan in March 2011.

\section{Evaluation of scanning approaches and methods}

The evaluation of the different approaches and methods faces several challenges. First, each of the methods described above has advantages and disadvantages depending on the specific circumstances under which they are applied, i.e. the interests of the client, the wider policy context which they are associated with, time and budget restrictions, capacities available or topic specific features like potential risks and urgency, depth and width of implications, degree of public awareness and concerns. Additionally, some methods are better for the initial phases of the scanning process, while others fit better into the analysis phase (cf. Table 2).

In this regard an evaluation across the different approaches and methods is difficult as their success is highly contextual. However, some common criteria can be identified if they reflect the information needs and interests of policy-makers and the degree to which they are met by the different tools and approaches (see Table 3).

A second challenge is that the two different approaches (exploratory scanning and issue-centred scanning) do not cover the whole scanning cycle but complement each other (cf. Fig. 1). In this regard, they are not directly comparable. They can be considered to be two departure points for the scanning activity. For example, the exploratory scanning approach represented by tools such as
Table 3. Evaluation criteria for scanning approaches and methods

Connections, clustering of weak signals and degree of relevance to a specific area

Duration of weakness of signal, also associated with time at which signal is observed

Origin (stakeholder(s) behind them) and novelty of weak signals

Rising ethical, legal, societal or cultural issues

Existence of a strategy already concerned with specific weak signal(s) and emerging issues by a government or industry, political party or lobby, or international organisation

Positive and negative impacts and associated policy implications

Policy recommendations

text-mining, Twitter or wiki scanning hardly goes beyond the second phase, i.e. processing of weak signals. On the other hand, in the focused expert review, the main scanning process of the issue-centred scanning, spans through all the different phases of the scanning cycle and can be assisted by other tools.

Any useful evaluation of different methods based on the information needs of policy-makers should apply to methods or combination of methods that cover all the different phases of the scanning activity. Following the framework of tools and methods presented in Fig. 2, certain combinations of methods can be created to provide a complete evaluation along the scanning process. Three possible combinations are defined below:

- Twitter/wiki scanning which is complemented by processing of weak signals.

- Focused expert review which is complemented by text-mining.

- Focused expert review which is assisted by experts' survey, literature review and attending conferences.

The first combination can be considered to be more in line with the exploratory scanning approach, while the last two refer mainly to the issue-centred approach. These combinations present different features in terms of the degree of automation of the tools that are used and the degree to which they depend on participatory tools bringing together experts' knowledge (cf. Table 4). Table 5 evaluates the three combined approaches based on the criteria mentioned in Table 3.

The SESTI experience of applying these methods (not necessarily as combined above) revealed that it is important to have several alternative methods and means to cover the needs of the different scanning phases as the same method may have advantages with respect to one criterion and scanning phase but disadvantages for another. Additionally, the SESTI experience has shown the crucial value of networks and human expertise. In general, across all combined tools the value of the 'human factor' outweighed the benefits of any automation tool as these are dependent on a human mind understanding, analysing and synthesising various pieces of information. 
Table 4. Description of three combined approaches

\begin{tabular}{|c|c|c|c|}
\hline & $\begin{array}{l}\text { (a) Semi-automated, participatory } \\
\text { approach }\end{array}$ & $\begin{array}{l}\text { (b) Semi-automated, non-participatory } \\
\text { approach }\end{array}$ & (c) Manual combined approach \\
\hline & $\begin{array}{l}\text { (Twitter/wiki }+ \text { processing of weak } \\
\text { signals) }\end{array}$ & (Focused expert review + text-mining) & $\begin{array}{l}\text { (Focused expert } \\
\text { review }+ \text { survey }+ \text { conferences })\end{array}$ \\
\hline Automation tools & $\begin{array}{l}\text { Search engines, automated news } \\
\text { aggregators, topic finders }\end{array}$ & $\begin{array}{l}\text { Text-mining based on proximity of } \\
\text { words and frequency of terms. } \\
\text { Search engines for focused expert review }\end{array}$ & Search engines \\
\hline $\begin{array}{l}\text { Size of source of } \\
\text { information }\end{array}$ & Limited: specific & Large: wide & $\begin{array}{l}\text { Limited: specific for survey and confer- } \\
\text { ences, large for focused expert review, } \\
\text { literature review }\end{array}$ \\
\hline $\begin{array}{l}\text { Duration: } \\
\text { observation time }\end{array}$ & $\begin{array}{l}\text { Through reports, blogs, groups, social } \\
\text { networks, Twitter, sites, etc. (observa- } \\
\text { tion time depends on participants) }\end{array}$ & $\begin{array}{l}\text { Reports as pdf, docs, rtf and parts } \\
\text { from website, e.g. RSS (Really Simple } \\
\text { Syndication) feeds for text-mining. } \\
\text { Articles, websites, videos for focused } \\
\text { expert review. Any other info } \\
\text { piece for second-round scanning } \\
\text { (observation time depends on scanner) }\end{array}$ & $\begin{array}{l}\text { Experts' knowledge; literature; } \\
\text { conferences for additional signal/issue } \\
\text { selection and refinement of reference } \\
\text { frame (observation time depends on } \\
\text { participants and scanner) }\end{array}$ \\
\hline $\begin{array}{l}\text { Signal/issue selection } \\
\text { criteria }\end{array}$ & $\begin{array}{l}\text { Pre-defined criteria based on sources } \\
\text { other futurists use (sense of credibil- } \\
\text { ity) and through interactions and } \\
\text { discussions within experts and } \\
\text { networks about implications }\end{array}$ & $\begin{array}{l}\text { Text-mining enables either 'bottom-up' } \\
\text { analysis (from 'scratch') or via } \\
\text { manually inserted keywords. Selection } \\
\text { of which documents to include is } \\
\text { crucial especially in 'bottom-up' } \\
\text { analysis. Focused expert review is } \\
\text { based on pre-defined reference frame }\end{array}$ & $\begin{array}{l}\text { Pre-defined criteria reflected in survey } \\
\text { questions, also driven by specific } \\
\text { reference framework used in focused } \\
\text { expert review. For literature review } \\
\text { and selection of conferences same } \\
\text { reference framework }\end{array}$ \\
\hline Verification method & Cross-referencing with other articles & Discussions with experts & $\begin{array}{l}\text { Discussions with experts; conferences; } \\
\text { further outlook in literature }\end{array}$ \\
\hline
\end{tabular}

Overall, the features of the participatory methods enabling verification and filtering of results as well as policy implications and recommendations are of crucial importance for the last stages of the scanning process. At the same time, semi-automated methods are appreciated, especially those drawing on large expert communities, as they enable systematic scanning and retrieval of 'inside' information in the first scanning phases as well as in verifying the results. The value of the more 'conventional' methods like expert surveys, literature review and conference visits is more relevant for targeted tasks requiring expert inputs like reviewing the state-of-the-art in a given field, searching for comprehensive reports on weak signals and emerging issues, and verifying the results or reference frames which may be required throughout all the scanning phases.

\section{Conclusion}

Horizon scanning is generally seen as an instrument with two main functions: the alerting function and the creative function. Horizon scanning alerts policy-makers to anticipate better and earlier emerging issues that will probably need their attention. At the same time, it is a creative instrument that can be used to reassemble issues or even create new emerging issues on the basis of the analysis and integration of scan data. This function allows a wider range of policy options to be considered. It also helps in the assessment of developed policies in relation to their resilience to unknown, but plausible, new emerging issues.

For the alerting function a comprehensive method is needed to scan and assess early warning signals that may indicate potential emerging issues from of a variety of media and sources. In the SESTI project this was done by internet scanning with the use of the publicly available search engines such as Google and Bing. Additionally, expert networks were invited through web-based tools to help in the search for early warning signals. For the more creative function this method needs to be complemented with tools and participative processes that, on one hand, may be focused on clustering and synthesis of scan data and, on the other hand, on human imagination and creativity. Within the SESTI project different text-mining techniques were used for this purpose as well as exercises which used participative web tools such as Wikipedia, blogs and Twitter.

The SESTI project also included workshops with experts and stakeholders that served both of the two main functions. On the one hand, alerts for emerging issues coming from the (expert reviewed) internet scanning were discussed and assessed, thus serving the alert function. On the other hand, the signals and issues presented were also used to evoke creative ideas by recombining (parts of the) identified issues, thus serving the creative function.

The SESTI experience provides insights on how to organise and implement exploratory and issue-centred horizon scanning processes to produce alerts but also to 
Table 5. Analysis of three combined approaches across evaluation criteria

(a) Semi-automated, participatory approach (b) Semi-automated, non-participatory approach

(c) Manual combined approach

(Focused expert review + text-mining)

\section{Medium}

Automatic clustering of keywords in the primary signal contains a rich narrative that already relates many

\section{Medium}

As attached to raw sources for via tracing back earlier signals and by monitoring secondary signals Medium in focused expert review findings

High

In focused expert review by tracing

High

In text-mining, the analyst can look deeper in case certain words can be connected. As reported in raw sources in focused expert review

Medium

In text-mining dependent on sources fed into database. In unstructured
Connection/ clustering

\section{Duration:}

observation time

Origins

Novelty

Filtering based on relevance, and credibility

(Twitter/wiki + processing of weak signals)

Medium

Connections pre-defined through tagging. Also inputs about links from field experts and futurists. Clusters are based on occurrence of similar words in text files and through attached criteria of collected weak signals.

Cross-checking with results from recent foresight exercises

High

As reported but also time series tracing possible in blogs, google etc.

High

As included/connected to info piece

High

Cross-checking with scientific databases. Cross-checking with results from recent foresight exercises

Ethical, legal, societal issues

Existing strategies and relevant stakeholders

Policy implications assessment
Medium

Expert discussions, futurists symposia, how/where newspaper articles are written. Further search for related documents dealing with ELSI dimensions

Medium

Through discussion with peers
Medium
As reported/criticised in raw resources.

In processing existing policies are identified and considered in framing/ reframing of potential issues by manual search for related previous foresight activities and related ongoing policy initiatives and discourses

Medium

Associated policy implications of text-mining. In focused expert review aspects of the emerging issues. Second scan may deliver richer or alternative/ contradictory descriptions that may lead to combined or meta descriptions text-mining. In focused expert review

As revealed by text-mining; as reported back on internet (e.g. google timeline) Internet text-mining results can be filtered according to their issue, to their organisation, to their reputation and creditability on the Internet. In focused expert review ensured through application of specific reference frame and associated selection criteria

Medium emerging issues are analysed by comparing emerging issues identified with topics in previous published thematic foresight report as well as in recently published policy documents
Text-mining can show policy-related terms appearing in a document and links between keywords and stakeholders. These cases require more examination by analyst. In focused expert review secondary scanning can reveal relevant information

\section{High}

Text-mining can show the policy related terms. In focused expert review narratives in the primary scanning usually contain already policy implications or even policy advice of author. While secondary scanning usually gives ideas on elaboration of proposed policies or of critics
(Focused expert review + survey + conferences)

High

Connections and clustering is made as reported in survey responses, literature and based on reviewers' expertise. Visit of conferences to help confirm findings and clustering

Medium

As reported by survey respondents; use of tools like google timeline in focused expert review; as reported in literature/discussed in conferences Medium

As reported by survey respondents; as reported in focused expert review findings; as reported in literature

High

As reported by survey respondents; use of tools like google timeline in focused expert review; as reported in literature/discussed in conferences High

As reported in survey responses, literature and raw sources in focused expert review; and as discussed at conferences

High

In survey ensured by survey questions. In focused expert review ensured through application of specific reference frame and associated selection criteria. In literature review based on documents selected for review based on experts' knowledge. In conferences through discussions with peers

High

As reported in survey responses and in literature and synthesised by reviewers. In focused expert review secondary scanning can reveal relevant information. Conferences are a place where existing strategies/ policies are usually discussed

High

As reported in survey responses and in literature. As facilitated by narratives in focused expert review. Conferences are useful to recruit potential policy workshop participants 
Table 5. Continued

(a) Semi-automated, participatory approach (b) Semi-automated, non-participatory approach
High

Meta descriptions of issues can be discussed in Workshops with experts and stakeholders and usually lead to recommendations
High

As reported in survey responses and in literature. As facilitated by policy workshops recommendations (c) Manual combined approach

\author{
ation of relevant thematic foresight \\ reports and recently published policy \\ documents \\ Medium
}

(1) 
model-based forecasting. In general, model-based forward-looking results are taken into account far more seriously by policy-makers than horizon scanning data, even though economic models completely failed to forecast the financial crisis of 2007-8, even in the shortest term. On the other hand, horizon scans in the Netherlands and UK had already spotted the financial crisis two years before it started. In the Netherlands this emerging issue was ranked among the top 20 most important issues. However, because of a shortage of resources to monitor these 20 issues in more depth, policy-makers downgraded the importance of the specific issue by maintaining their faith in the reassuring messages from the economic planning agencies (van Rij 2010b).

The special nature of horizon scanning activities points to the importance of continuity to increase the relevance, validity and timeliness of the information. This implies sustaining resources. As Choo (2001) points out, horizon scanning is a long-term investment that needs a critical mass of talent and resources. Nevertheless, the results may still not be taken up by policy-makers for a number of reasons as mentioned above. Involving policy-makers sufficiently early in the process so that they can provide regular information on the process and obtain results is important. It is also important that policy-makers are actively engaged, especially in interpreting the results and deriving policy recommendations. Even more so, it is crucial to make them aware of the benefits of effectively dealing with potentially important issues early enough as well as the risks of overlooking them.

\section{Acknowledgements}

The views expressed are purely those of the author and may not in any circumstances be regarded as stating an official position of the European Commission.

\section{Notes}

1. See <http://www.bis.gov.uk/foresight/our-work/ horizon-scanning-centre $>$ accessed 21 December 2011.

2. See <http://app.hsc.gov.sg/public/www/home.aspx> accessed 8 September 2011.

3. See <http://sesti.info/> accessed 8 September 2011.

4. No use was made from more advanced tools as web-crawler or spyware.

5. See <http://news.google.com/news/advanced_news_ search $? \mathrm{pz}=1 \& \mathrm{cf}=$ all\&ned $=\mathrm{us} \& \mathrm{hl}=\mathrm{en}>$ accessed 21 December 2011.

6. See <http://www.google.com/insights/search/> accessed 21 December 2011.

7. See < http://www.bing.com/> accessed 21 December 2011.

8. See <http://twitter.com/> accessed 21 December 2011.
9. Provided by Thomson Reuters <http:// thomsonreuters.com/products_services/science/ science_products/a-z/web_of_science/> accessed 21 December 2011.

10. See <http://www.gapminder.org/> accessed 21 December 2011.

\section{References}

Agarwal, N., Galan, M., Liub, H. and Subramanyab, S. (2010) 'WisColl: Collective wisdom based blog clustering', Information Sciences, 180: 39-61.

Boden, M., Cagnin, C., Carabias, V., Haegeman, K. and Könnölä, T. (2010) 'Facing the future: Time for the EU to meet global challenges', 24364 EN, 6/2010. Luxembourg: Office for Official Publications of the European Commission.

Böschen, S., Kastenhofer, K., Rust, I., Soentgen, J. and Wehling, P. (2010) 'Scientific nonknowledge and its political dynamics: The cases of agri-biotechnology and mobile phoning', Science Technology and Human Values, 35: 783-811.

Botterhuis, L., van der Duin, P., de Ruijtera, P. and van Wijck, P. (2010) 'Monitoring the future. Building an early warning system for the Dutch Ministry of Justice', Futures, 42: 454-65.

Brohee, S., Barriot, R. and Moreau, Y. (2010) 'Biological knowledge bases using Wikis: combining the flexibility of Wikis with the structure of databases', Bioinformatics, 26: 2210-1.

Chilton, P. A. (2004) 'Analyzing Political Discourse: Theory and Practice'. London: Routledge.

Choo, C. W. (2001) 'Environmental scanning as information seeking and organizational learning', Information Research, $7 / 1<$ http://choo.fis.utoronto.ca/IR/choo/choo.html > accessed 30 September 2011.

Czaplicka-Kolarz, K., Stanczyk, K. and Kapusta, K. (2009) 'Technology foresight for a vision of energy sector development in Poland till 2030. Delphi survey as an element of technology foresighting', Technological Forecasting and Social Change, 76: 327-38.

Freund, F. (2011) 'Pre-earthquake signals: Underlying physical processes', Journal of Asian Earth Sciences, 41: 383-400.

Georghiou, L. (2007) 'Future of foresighting for economic development', UNIDO, Vienna. <http://www.unido.org/ foresight/rwp/dokums_pres/tf_plenary_georghiou_201.pdf > accessed 8 September 2011.

Hiltunen, E. (2008a) 'The future sign and its three dimensions', Futures, 40: 247-60.

- (2008b) 'Good sources of weak signals: A global study of where futurists look for weak signals', Journal of Futures Studies, 12: 21-42.

Könnölä, T., Salo, A., Cagnin, C., Carabias, V. and Vilkkumaa, E. (2012) 'Facing the future: Scanning, synthesizing and sense-making in horizon scanning', Science and Public Policy, 39: $\mathrm{XXX}-\mathrm{XXX}$, in this special issue.

Loveridge, D. (2009) 'Foresight: The Art and Science of Anticipating the Future'. New York and London: Routledge.

Mandavilli, A. (2011) 'Trial by Twitter', Nature, 469/7330: 286-7.

Marvin, H. J. P., Kleter, G. A., Frewer, L. J., Cope, S. et al. (2009) 'A working procedure for identifying emerging food safety issues at an early stage: Implications for European and international risk management practices', Food Control, 20: $345-56$.

Mendonça, S., Pina e Cunha, M., Ruff, F. and Kaivo-oja, J. (2009) 'Venturing into the wilderness: Preparing for wild 
cards in the civil aircraft and asset-management industries', Long Range Planning, 42: 23-41.

Mons, B., Ashburner, M., Chichester, C., van Mulligen, R. et al. (2008) 'Calling on a million minds for community annotation in WikiProteins', Genome Biology, 9: 5.

Morrison, J. L. (1992) 'Environmental scanning'. In: Whitely, M. A., Porter, J. D. and Fenske, R. H. (eds) A Primer for New Institutional Researchers, pp. 86-99. Tallahassee, FL: Association for Institutional Research.

Pang, A. S.-K. (2010) 'Social scanning: Improving futures through Web 2.0; or, finally a use for Twitter', Futures, 42: 1222-30.

Saritas, O. and Smith, J. E. (2011) 'The Big Picture: Trends, drivers, wild cards, discontinuities and weak signals', Futures, 43: 292-312.

Smith, J., Cook, A. and Packer, C. (2010) 'Evaluation criteria to assess the value of identification sources for horizon scanning', International Journal of Technology Assessment in Health Care, 26: 348-53.

Stein, L. D. (2008) 'Towards a cyberinfrastructure for the biological sciences: progress, visions and challenges', Nature Reviews Genetics, 9: 678-88.

Sutherland, W. J., Clout, M., Côté, I., Daszak, P. et al. (2010) 'A horizon scan of global conservation issues for 2010', Trends in Ecology and Evolution, 25: 1-7.
Sutherland, W. J., Fleishman, E., Mascia, B., Pretty, J. and Rudd, M. (2011) 'Methods for collaboratively identifying research priorities and emerging issues in science and policy', Methods in Ecology and Evolution, 2: 238-47.

Uskali, T. (2005) 'Paying attention to weak signals: The key concept for innovation journalism', Innovation Journalism, 2/11 $<$ www.innovationjournalism.org $>$ accessed 13 March 2012.

Van der Steen, M. (2008) 'Ageing or silvering? Political debate about ageing in the Netherlands', Science and Public Policy, 35: 575-83.

Van Dijk, T. A. (2002) 'Political discourse and political cognition'. In: Chilton, P. and Schäffner, C. (eds) Politics as Text and Talk: Analytic Approaches to Political Discourse, pp. 203-31. Philadelphia, PA: John Benjamins.

Van Rij, V. (2010a) 'Joint horizon scanning: identifying common strategic choices and questions for knowledge', Science and Public Policy, 37: 7-18.

. (2010b) 'Horizon scanning: Monitoring plausible and desirable futures'. In: In't Veld, R. J. (ed.) Knowledge Democracy: Consequences for Science, Politics, and Media, pp. 227-240. Berlin/Heidelberg: Springer.

. (2012) 'Wild cards as future shakers and shapers'. In: Giaoutzi, M. and Sapio, B. (eds) Recent Developments in Foresight Methodologies. New York: Springer Verlag. 